\title{
ESTADO CONSTITUCIONAL DE DERECHO Y PONDERACIÓN: HACIA LA SUPERACIÓN DE LA FALSA DISYUNTIVA ENTRE LIBERTAD Y SATISFACCIÓN DE LOS DERECHOS SOCIALES FUNDAMENTALES*
}

\author{
CONSTITUTIONAL STATE OF LAW AND \\ WEIGHTING: THROUGH THE OVERCOMING \\ OF THE FAKE JUNCTURE BETWEEN \\ FREEDOM AND SATISFACTION OF THE \\ FUNDAMENTAL SOCIAL RIGHTS
}

\author{
J. Ignacio Núñez Leiva** \\ Fecha de recepción: 20 de agosto de 2013 \\ Fecha de aprobación: $1^{\circ}$ de octubre de 2013 \\ Disponible en linea: 30 de junio de 2014
}

\section{Para citar este artículo / To cite this article}

J. Ignacio Núñez Leiva, Estado constitucional de derecho y ponderación: Hacia la superación de la falsa disyuntiva entre libertad y satisfacción de los derechos sociales fundamentales, 128 Vniversitas (2014). http://dx.doi. org/10.11144/Javeriana.VJ128.ecdp

doi:10.11144/Javeriana.VJ128.ecdp

* El presente artículo es producto del trabajo en el área de investigación de la facultad de derecho de la Universidad Finis Terrae, Chile.

** Abogado. Licenciado en Derecho por la Pontificia Universidad Católica de Chile. Diplomado en Derechos Humanos por la Universidad Católica del Uruguay. Post Graduado en Derecho por la Universidad de Castilla La Mancha, España. Especialista en Constitucionalismo y Garantísmo (2009) y Especialista en Justicia Constitucional y Procesos Constitucionales (2012). Magister en Derecho Público por la Pontificia Universidad Católica de Chile. Diploma de Estudios Avanzados y Doctor (c) en Derecho por la Universidad de Castilla La Mancha. Investigador, Facultad de Derecho Universidad Finis Terrae, Chile. Correo electrónico: jinunez@uc.cl; jinunez@uft.cl 


\section{RESUMEN}

El presente trabajo analiza los derechos sociales en el contexto del Estado constitucional de derecho y propone el uso de la ponderación para superar la falsa dicotomía entre libertad y derechos sociales.

Palabras clave: Estado constitucional de derecho; derechos sociales 


\section{ABSTRACT}

This paper analyzes social rights in the context of contemporary constitutionalism and proposes the use of the proportionality tests overcome the false dichotomy between freedom and social rights.

Keywords: Constitutional rule of law; social rights

\section{SUMARIO}

INTRODUCCIÓN.- I. UN PROLOGÓMENO TERMINOLÓGICO.- II. EL ESTADO CONSTITUCIONAL DE DERECHO: EL ESCENARIO DE LA PONDERACIÓN.- II. EL ESTADO CONSTITUCIONAL DE DERECHO: EL ESCENARIO DE LA PONDERACIÓN.- III. LA PONDERACIÓN DE DERECHOS SOCIALES.- CONCLUSIONES Y REFLEXIONES FINALES. 


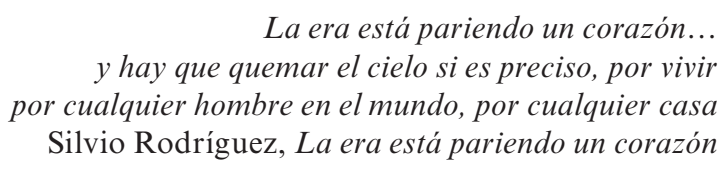

\section{INTRODUCCIÓN}

Este trabajo tiene como propósito contextualizar la figura de la ponderación en el escenario que le ha servido, al mismo tiempo, de origen y espacio fecundo para su desarrollo: el Estado constitucional de derecho.

Este ejercicio de geografía conceptual no obedece a un mero afán teórico sino a un objetivo concreto: afirmar que dentro de tal panorama los denominados derechos sociales - al igual que todo derecho fundamental - dejan de ser considerados disposiciones programáticas para ser estimados como normas especiales con el carácter de principios o directrices, factor que determina su obligatoriedad para todos los sujetos normativos de las Constituciones y que sugiere el uso de la ponderación como técnica para su aplicación concreta, particularmente ante potenciales conflictos con otros derechos fundamentales. Se propone finalmente aprovechar la estructura de principios que actualmente ostentan los derechos fundamentales y la alternativa de la ponderación, no únicamente para resolver conflictos de derechos, sino también para dar lugar a un argumento defendido por Robert Alexy, y de alguna manera también por Garzón Valdés y Luis Prieto, útil para eludir la artera y artificial idea que sugiere la existencia de una dicotomía irreconciliable entre la protección de la libertad y los derechos sociales fundamentales.

\section{UN PROLOGÓMENO TERMINOLÓGICO}

El supuesto carácter prestacional de los denominados derechos sociales es uno de sus rasgos más frecuentemente subrayados, tal vez porque, desde el punto de vista jurídico, resulta más explicativo o definidor que aquellos otros que se basan en consideraciones históricas, ideológicas o sociológicas. El criterio delimitador de tal adjetivo residiría - según el maestro Prieto Sanchís- en el contenido de la obligación que, usando terminología kelseniana, 
constituye el reflejo del derecho: en los derechos civiles o individuales, el contenido de la obligación consiste en una abstención u omisión, en un no hacer nada que comprometa el ejercicio de la libertad o el ámbito de inmunidad garantizado; en cambio, en los derechos sociales el contenido de la obligación es de carácter positivo, de dar o de hacer ${ }^{1}$.

Con todo, si consideramos que en estricto rigor un derecho prestacional es aquel en cuya virtud se asegura a su titular algo que, si tuviese medios económicos suficientes y se encontrase disponible en el mercado, podría adquirir por sí mismo de particulares ${ }^{2}$, la idea de identificarlo automáticamente con los derechos sociales es inconveniente por las siguientes razones.

El primer problema de asimilar a niveles de esencialidad los derechos sociales con los derechos prestacionales, consiste en que al hacerlo un número no menor de importantes derechos tradicionalmente considerados sociales - en su dimensión teleológica, esto es su funcionalidad para alcanzar la igualdad sustancial - resultarían excluidos debido a que no incluyen en su contenido ninguna clase de prestación o al menos no lo hacen en sentido estricto. Tal es el caso de todos los que expresan restricciones a la autonomía individual en el contrato de trabajo: como la limitación de jornada, un salario mínimo, las vacaciones anuales, o el derecho a huelga y el derecho a sindicación. En segundo lugar - siguiendo nuevamente a Prieto-cuando hablamos de derechos prestacionales en sentido estricto nos referimos fundamentalmente a bienes o servicios económicamente evaluables: subsidios de desempleo, enfermedad o vejez, sanidad, educación, vivienda, etc. De otro modo todos los derechos fundamentales -incluso los no sociales - merecerían el rótulo de prestacionales, dado que todos ellos exigen en mayor o menor medida una organización estatal que permita su ejercicio o que los defienda frente a intromisiones ilegítimas ${ }^{3}$. Desde la tutela judicial efectiva hasta el derecho de sufragio, pasando por denominado derecho a defensa jurídica técnica, casi todos los derechos requieren de un dar o hacer estatal, esto es de prestaciones en sentido amplio.

1 Luis Prieto Sanchís, Los derechos sociales y el principio de igualdad sustancial, Revista del Centro de Estudios Constitucionales, No. 22, 9-56 (1995). Pág. 15.

2 Robert Alexy, Teoría de los derechos fundamentales, Centro de Estudios Políticos y Constitucionales, Madrid, 391 (2008).

3 Prieto, óp. cit., 15. 
En tercer lugar, conviene advertir que las técnicas prestacionales no pertenecen en exclusiva a alguna clase de derechos, sino que en general son aplicables a cualquiera de los fines del Estado, incluso a los derechos civiles y políticos. Prieto se ha referido a esto en su obra Curso de derecho eclesiástico acudiendo al caso de la libertad religiosa que, según una opinión difundida, no solo ha de ser respetada, sino también protegida y hasta subvencionada a fin de que su ejercicio pueda resultar verdaderamente efectivo ${ }^{4}$. Otros ejemplos pueden estar constituidos por los derechos políticos que resultan subvencionados mediante prestaciones en los Estados en donde existe financiamiento público de partidos políticos o por la libertad de expresión que suele estimularse a través de la existencia - no monopólica - de medios de comunicación pertenecientes al Estado.

Por otra parte, y a consecuencia de lo anterior, en términos argumentativos asimilar automáticamente a derechos sociales con derechos prestacionales deja injustificadamente abierto un flanco de crítica para los derechos sociales que no suponen prestación alguna. Y lo hacen justamente ante el argumento más difundido en contra la eficacia de los derechos sociales: que la satisfacción de los derechos prestacionales depende de la potencia de la hacienda pública.

Finalmente, la identificación a priori de los derechos sociales con derechos de prestación en sentido estricto, envuelve una paradoja. En general los derechos prestacionales requieren, para ser reconocidos como tales, de una suficiente determinación de sus titulares, contenidos y sujetos pasivos, además de la asignación de una garantía jurisdiccional. Sin embargo, justamente uno de los factores de aparente debilidad de los derechos fundamentales sociales está constituido por la apretura de su supuesto de hecho, la sub-determinación de sus consecuencias jurídicas y la dificultad para dispensarles tutela judicial. Es decir, de alguna manera hablar sin más de derechos sociales como derechos prestacionales en sentido estricto importa confundir su configuración positiva real con su estatuto deseado. Con todo, como veremos a continuación, la apertura o indeterminación de las normas constitucionales no

4 Prieto, óp. cit., 16. 
es óbice para su obediencia en calidad de normas jurídicas plenas en el Estado constitucional de derecho.

\section{EL ESTADO CONSTITUCIONAL DE DERECHO: EL ESCENARIO DE LA PONDERACIÓN}

En el plano teórico, el constitucionalismo contemporáneo tiene el afán de describir y destacar los logros de la constitucionalización, es decir de aquel proceso causado por una revitalizada Constitución que ha ocasionado una modificación de los sistemas jurídicos respecto de lo existente antes del despliegue del proceso mismo y que deviene en el Estado constitucional de derecho.

Según Prieto Sanchís, estos conceptos sirven para designar un modelo teórico, pero también para referirse a una forma de organización política que se viene abriendo paso desde mediados del siglo XX, que no necesariamente reúne en todas sus manifestaciones signos de uniformidad, sino unos rasgos esenciales que permiten hablar de algo diferente, no radicalmente nuevo, pero sí distinto a lo que pudo representar el Estado de derecho decimonónico ${ }^{5}$.

El cambio mencionado se produce a partir de la introducción de conceptos como el valor normativo de la Constitución y la vinculación de la jurisdicción a los derechos fundamentales ${ }^{6}$. Ello es el corolario de la fusión de dos tradiciones, la de las Constituciones materiales; aquellas que presentan un denso contenido sustantivo integrado por normas de diferente valoración (valores, principios, derechos, directrices) y las Constituciones garantizadas, caracterizadas porque su protección o efectividad se encomienda a jueces o, si se prefiere, en su sistema existen normas secundarias, de organización y procedimiento, destinadas a depurar o sancionar toda infracción de las normas sustantivas o relativas a derechos. Así la Constitución se convierte en límite y norma directiva fundamental. Frente al rousseauniano poder constituyente que nunca termina de constituirse — nos dice Prieto - y que desemboca en la superioridad del parlamento y de su ley — lógica básica de los

5 Luis Prieto Sanchís, Justicia constitucional y derechos fundamentales, Trotta, Madrid, 107 (2003)

6 Alexei Julio Estrada, Los tribunales constitucionales y la eficacia entre particulares de los derechos fundamentales en Miguel Carbonell, ed., Teoría del neoconstitucionalismo, ensayos escogidos, 121-148, Trotta, Madrid, 148 (2007). 
sistemas de raíz francesa- el poder constituyente toma cuerpo en un texto jurídico supremo, válido, vigente y garantizado ${ }^{7}$. Todo lo anterior, agregaría Manuel Aragón, ha sido producto de adicionar al concepto liberal de constitución la impronta social y democrática de derecho reconociendo su valor de norma jurídica ${ }^{8}$.

El resultado de esta combinación, señala con contundencia Ferrajoli, es un nuevo modelo de derecho y democracia: el Estado constitucional de derecho, que es fruto de un verdadero cambio de paradigma respecto al modelo paleo-positivista del Estado legislativo de derecho ${ }^{9} \mathrm{y}$ en el cual la Constitución es fuente del derecho en un triple sentido: primero, por contener normas, segundo, por disponer parámetros sustantivos y procedimentales para abrogar y/o invalidar normas inferiores preconstitucionales e invalidar normas posteriores que la contravengan, y tercero — siendo quizás esto lo más destacable - por entenderse que las normas constitucionales son idóneas para disciplinar directamente no solo la organización estatal y las relaciones entre el Estado y las personas, sino también las que ocurran entre estas últimas, y son por tanto susceptibles de ser aplicadas por cualquier juez y no solo por el juez constitucional.

Todo lo anterior claramente altera el paradigma decimonónico del Estado de derecho y afecta las bases del sistema de fuentes elaborado a su amparo, las de su teoría constitucional y del derecho, y su concepto de democracia.

Con respecto al sistema de fuentes, la Constitución irrumpe como fuente del derecho en el sentido pleno de la expresión, es decir, como origen inmediato de derechos y obligaciones, y no solo como fuente de las fuentes. Ello altera la relación Constitución-ley. De una dinámica en la cual los derechos y obligaciones contenidos en la Constitución adquirían fuerza en tanto eran desarrollados por la ley, se pasa a una relación de subordinación en donde —en palabras de Susanna Pozzolo - la ley opera como un mero factor de mediación entre las exigencias constitucionales y las exigen-

7 Luis Prieto Sanchís, El constitucionalismo de los derechos, en Miguel Carbonell, ed., Teoría del neoconstitucionalismo, ensayos escogidos, 213-236, Trotta, Madrid, 214 (2007).

8 Manuel Aragón Reyes. La constitución como paradigma en Miguel Carbonell, ed., Teoría del neoconstitucionalismo, ensayos escogidos, 29-40, Trotta, Madrid, 35 (2007).

9 Luigi Ferrajoli, Sobre los derechos fundamentales, en Miguel Carbonell, ed., Teoría del neoconstitucionalismo, ensayos escogidos, 71-90, Trotta, Madrid, 71 (2007). 
cias prácticas ${ }^{10}$. Lo cual, de paso, nos obliga además a repensar el principio dinámico con el que por tanto tiempo se caracterizó el sistema jurídico, pues la innovación por parte de normas subconstitucionales, de acuerdo a esta nueva lógica, es válida en tanto su contenido sea deudor de los principios y valores contenidos en la Constitución, rasgo propio de los sistemas estáticos ${ }^{11}$.

En síntesis, la Constitución se instala como aquel derecho por sobre el derecho y opera como una convención sobre el coto vedado $^{12}$, o acerca de lo que es indecidible para cualquier mayoría ${ }^{13}$.

Esto último es efecto de lo que Prieto Sanchís denomina "rematerialización constitucional", factor que resulta clave para entender la existencia de este nuevo derecho (el constitucional) que se ubica por sobre el resto del derecho (principalmente el legal) ${ }^{14}$. Las Constituciones actuales están dotadas no solo de normas formales, de competencia o procedimiento, dirigidas a orientar el ejercicio de los poderes estatales y la relación entre los mismos, sino también - y sobre todo- de normas sustantivas que pretenden trazar límites negativos y vínculos positivos a lo que dichos poderes están en condiciones de decidir legítimamente. Siguiendo al mismo autor, justamente aquello que Kelsen temía es el sello de identidad de este nuevo derecho: gira en torno y se subordina a derechos, valores, principios y directrices ${ }^{15}$.

En efecto, estas nuevas piezas fundamentales del derecho son normas "muy generales", "redactadas en términos particularmente vagos", son "programáticas" en el sentido de que imponen la obligación de perseguir determinados fines, "expresan los valores superiores del ordenamiento jurídico" y sirven como "criterio de interpretación del derecho subconstitucional", por mencionar solamente algunos de los sentidos o características con que según Atienza y Ruiz Manero se les ha atribuido ${ }^{16}$.

10 Susana Pozzolo, Neoconstitucionalismo y especificidad de la interpretación constitucional, Doxa, No. 21, 339-353 (1998). Pág. 341.

11 Alfonso García Figueroa, Principios y positivismo jurídico, El no positivismo principialista en las teorías de Ronald Dworkin y Robert Alexy, Centro de Estudios Políticos y Constitucionales, Madrid, 62 (1998).

12 Ernesto Garzón Valdés, Algo más acerca del coto vedado, Doxa, No. 6, 209-213 (1989).

13 Luigi Ferrajoli, Derecho y razón. Teoría del Garantísmo penal, Trotta, Madrid, 858 (1998).

14 Prieto, óp. cit., 119 (2007).

15 Prieto, óp. cit., 215 (2007).

16 Manuel Atienza \& Juan Ruiz, Las piezas del derecho, Ariel, Barcelona, 4 (1996). 
Tal vez, los elementos más importantes en estas nuevas Cartas Fundamentales sean los principios y las directrices. Los primeros se caracterizan por estar formulados como enunciados que correlacionan siempre casos con la calificación normativa de una determinada conducta, pero de manera abierta. A diferencia de las reglas, carecen de una condición de aplicación determinada. No se trata de que posean una condición de aplicación con una periferia más o menos amplia, sino de que tales condiciones no se encuentran ni siquiera genéricamente determinada ${ }^{17}$. Las segundas se distinguen porque no solo sus condiciones de aplicación son abiertas o aplicables a - prácticamente - infinitos supuestos de hecho sino porque además su consecuencia jurídica (su modelo de conducta prescrita) tampoco está determinada ${ }^{18}$.

En lo relativo a la teoría constitucional, las relaciones recíprocas entre las funciones del Estado se alteran sustantivamente. El esquema decimonónico en el cual el legislador se encuentra investido con la capacidad de representar a la soberanía y por tanto invocar su inviolabilidad, resulta alterado por la aparición de una jurisdicción, ya sea ordinaria o específicamente constitucional, capaz de oponerse al contenido sustancial de sus decisiones, sea impidiendo que se conviertan en derecho vigente o suprimiéndolas del sistema jurídico con efecto general o particular según sea el caso, encargada de un continuo adecuamiento de la legislación a la Constitución mediante la tutela de las exigencia de la justicia sustancial en cada caso concreto y que se erige como el principal agente racionalizador del sistema jurídico ${ }^{19}$. Una función muy difícil de encuadrar en la formulación tripartita de la separación de poderes, e incluso en ciertos casos contra-mayoritaria.

Por su parte, la democracia operativa dentro del paradigma paleopositivista - citando a Ferrajoli - se ha convertido en democracia constitucional. Este es un modelo que no se limita a programar solo las formas de producción del derecho mediante normas procedimentales sino que además programa sus contenidos. Está constituido por normas meta-legales que disciplinan la máxima expresión de las mayorías contingentes presentes en el proceso de

17 Atienza \& Ruiz, óp. cit., 9 (1996).

18 Atienza \& Ruiz, óp. cit., 10 (1996).

19 Pozzolo, óp. cit., 342 (1998). 
generación de las leyes. En síntesis, la Constitución es aquel derecho por sobre el derecho y opera como una convención sobre el coto vedado ${ }^{20} \mathrm{o}$ acerca de lo que es indecidible para cualquier mayoría ${ }^{21}$.

La constitucionalización rígida de los derechos fundamentales nos sugiere el célebre jurista de Florencia - impone obligaciones y prohibiciones a los poderes públicos y ha insertado en la democracia una dimensión sustancial. Si las normas formales de la Constitución, esto es aquellas que disciplinan la organización de los poderes públicos, aseguran la dimensión formal de la democracia política, aquellas que establecen los principios y derechos fundamentales garantizan lo que se puede denominar democracia sustancial, y se refieren a qué no puede ser decidido o debe ser decidido por toda mayoría, vinculando toda la legislación bajo pena de invalidez a los derechos, principios y valores fundamentales. Ello cambia también la relación entre derecho y política, pues ya no es el primero quien se subordina a la segunda, sino a la inversa.

Así, el contexto del Estado Constitucional de Derecho, la condición - el espíritu del tiempo en que vivimos - expresa Zagrebelsky, deambula no entre uno sino entre varios principios o valores que configuran la convivencia colectiva: la libertad versus las reformas sociales, la igualdad genérica versus las legítimas diferencias en los casos concretos, la protección de los derechos individuales, pero también la de los sociales ${ }^{22}$. Si cada valor se entendiese como absoluto, sería imposible admitir la coexistencia de unos junto a otros. Si cada valor fuere a priori determinado o determinable, no existiría aquella tendencial incompatibilidad entre ellos mismos y entre los diversos proyectos que albergan.

Tan significativa ha sido la irrupción —o la detección- de los principios en las Constituciones que algún reputado jurista como Gustavo Zagrebelsky se atreve a afirmar que los principios serían normas propiamente constitutivas o constitucionales, mientras que las reglas - aun cuando se encuentren en la Constitución - no serían más que leyes reforzadas: a las reglas, por su simpleza, se las obedece, a los principios, se les presta adhesión ${ }^{23}$.

20 Ernesto Garzón Valdés, Algo más acerca del coto vedado, Doxa, No. 6, 209-213 (1989).

21 Luigi Ferrajoli, Derecho y razón. Teoría del garantismo penal, Trotta, Madrid, 858 (1998).

22 Gustavo Zagrebelsky, El derecho dúctil. Ley, derechos, justicia, Trotta, Madrid, 16 (2008).

23 Zagrebelsky, óp. cit., 110 (2008). 
El reconocimiento de los derechos fundamentales sociales en las cartas fundamentales del constitucionalismo contemporáneo enfatiza la dimensión objetiva de aquellos en cuanto que normas objetivas, las cláusulas que recogen derechos sociales vinculan a todos los poderes públicos, incluido el legislador, por lo que, en principio, nada impide que sean invocados en cualquier instancia jurisdiccional $\mathrm{y}$, por supuesto, que sirvan de parámetro para el juicio de constitucionalidad. Böckenförde ha resumido esa vinculación efectiva en tres aspectos: el fin o programa que supone un derecho social se sustrae a la en otro caso libertad del legislador; es inadmisible la inactividad o la desatención evidente y grosera por parte de los poderes públicos; y, por último, la satisfacción conferida a un derecho prestacional, una vez establecida, se muestra relativamente irreversible, en el sentido de que está protegida frente a una supresión definitiva o frente a una reducción que traspase los límites hacia la desatención grosera ${ }^{24}$.

\section{LA PONDERACIÓN DE DERECHOS SOCIALES}

La particularidad (funcional) de los principios estaría dada en que mientras las reglas, normas binarias, altamente determinadas en sus condiciones de aplicación y consecuencias jurídicas, proporcionan pautas nítidas acerca de cómo debemos, no debemos o podemos actuar, los principios no nos dicen nada a este respecto, pero sí nos entregan criterios para adoptar posiciones ante situaciones concretas, pero que en principio no aparecen de manera evidente contenidas en su espectro normativo ${ }^{25}$. Los principios no tienen eficacia en abstracto, requieren de casos concretos para ser operativos ${ }^{26}$.

En este contexto, una jerarquía de valores (o principios y directrices) resulta inútil e insuficiente ${ }^{27}$. Si así ocurriere existiría una incompatibilidad entre la Constitución y el carácter pluralista de la sociedad. En caso de conflicto, el principio o valor de mayor rango privaría de valor a todos los principios (constitucionales) inferiores y daría lugar a una amenazante tiranía de los valores, esencialmente

\footnotetext{
24 Luis Prieto Sanchís, Ley, principios, derechos, Dikynson, Madrid, 99 (1998).

25 Zagrebelsky, óp. cit., 110 (2008).

26 José Juan Moreso, Constitución: un modelo para armar, Marcial Pons, Madrid, 233 (2009).

27 Zagrebelsky, óp. cit., 124 (2008).
} 
destructiva. El pluralismo - la multiplicidad de principios vertebradores de diversos proyectos morales y políticos - y la consecuente ausencia de una preferencia respecto de algunos de ellos (de una jerarquía formal) exige que no exista, parafraseando a Zagreblesky, una ciencia sobre su articulación sino prudencia en su aplicación ${ }^{28}$.

Quizás la principal novedad o propuesta metodológica surgida bajo el Estado constitucional de derecho sea el famoso principio de proporcionalidad (también llamado método de la ponderación), instituto sobre el cual no ahondaremos pues sus perfiles son de amplio conocimiento. Con todo destacaremos que su aplicación, si bien no asegura una y solo una respuesta para cada caso concreto impone al sentenciador, aunque sea de manera perfectible, la necesidad de fundamentar sus decisiones a la hora de resolver un conflicto normativo que no admite la aplicación de las clásicas herramientas de solución de antinomias ${ }^{29}$, pues normalmente acontece entre disposiciones que "dicen mucho al legislador" pero "muу poco al juez"30.

En un modelo donde la aplicación del derecho no depende de procedimientos provistos únicamente de lógica formal y con aplicación automática y en el cual corresponde al sentenciador la búsqueda del derecho no solo en las normas de procedencia estatal - orientado permanentemente por la consideración y ponderación de los intereses sociales-, el rol de la judicatura no puede ser concebido simplemente como el de un ejecutor de las prescripciones genéricas contenidas en las normas emanadas del legislador.

En un contexto donde el derecho ha de verse como una práctica social compleja, consistente en decidir casos, justificar aquellas decisiones y producir normas derivadas de preceptos altamente abstractos, el ordenamiento jurídico no es un dato dado de antemano (a la espera de que un jurista teórico o un juez lo sistematicen), sino una actividad en la que teóricos y jueces participan ${ }^{31}$, y no desde afuera.

28 Zagrebelsky, óp. cit., 125 (2008).

29 Luis Prieto Sanchís, Neoconstitucionalismo y ponderación judicial, en Miguel Carbonell, Neoconstitucionalismos, Trotta, Madrid, 152 (2005).

30 Prieto, óp. cit., 217 (2007).

31 Manuel Atienza \& Juan Ruiz, Para una teoría postpositivista del derecho, Palestra, Lima, 152 (2009). 
Por eso, Zagreblesky afirma que el carácter de la actividad jurisdiccional, que se desenvuelve entre los principios y las reglas, entre los hechos y el derecho, debería conducir a la pregunta de si no resulta incongruente la gestión de un derecho ya no solo estatal bajo un modelo estatista que trata al funcionario judicial como un mero administrador, un burócrata lleno de apatía social, encargado de una aplicación exacta y geométrica de la ley, distante de toda influencia. El punto sobre el que sugiere insistir es en "la posición dual que necesariamente corresponde a los jueces en el Estado Constitucional: una especialísima y dificilísima posición de intermediación entre el Estado (como poder político-legislativo) y la sociedad (como sede de los casos que plantean pretensiones en nombre de los principios constitucionales), que no tiene paralelo en ningún otro tipo de funcionarios públicos" ${ }^{\prime \prime 2}$.

En este contexto, y a propósito de los derechos sociales, Luis Prieto afirma que las desigualdades inmerecidas deben ser compensadas mediante la adopción de medidas - regulaciones o prestaciones - pues, en palabras de Kymlicka, "las porciones distributivas no debieran estar influidas por factores que son arbitrarios desde el punto de vista moral". Es obvio que no toda diferencia debe combatirse; al contrario, algunas deben tolerarse y hasta tutelarse. Como escribe Ferrajoli, "el principio (o deber) de tolerancia sirve para fundar el conjunto de los derechos de libertad, pero además debe hablarse de un principio (o deber) de no tolerancia, que vale para fundamentar el concepto de los derechos sociales: aquello que está en la base de los derechos civiles, creencias y planes de vida, debe ser tolerado; aquello otro que está en la base de los derechos sociales, carencias o pobreza, no debe tolerarse" 33 .

En el mismo sentido - sumándose a la solución ponderativa de Alexy que enseguida revisaremos-Garzón Valdés, propone que el argumento principal a favor de los derechos sociales es la satisfacción del principio de la dignidad humana. Así lo hace cuando se pregunta ¿No podría suponerse que la no concesión de derechos sociales conduce a la exclusión de una buena parte de la ciudadanía, es decir a una sociedad indecente? ${ }^{34}$

32 Zagrebelsky, óp. cit., 149 (2008).

33 En Prieto, óp. cit., 91 (1998).

34 Ernesto Garzón Valdés, Cinco consideraciones acerca de la concepción de derechos sociales de 
Finalmente, Robert Alexy (desde una óptica incluso más precaria, pues la Ley Fundamental alemana no consagra directamente derechos de esta índole, sino que han sido jurisprudencialmente construidos en base al derecho general de igualdad) propone un modelo general justificativo de la satisfacción de los derechos fundamentales sociales basado en dos pilares: la ponderación y la superación de la falsa dicotomía libertad-igualdad.

Para el iusfilósofo alemán, los derechos fundamentales - todosson posiciones que, desde el punto de vista constitucional, son tan importantes que su atribución o denegación no puede quedar en manos de la mayoría parlamentaria. Esto implica que a cada uno le corresponden las posiciones jurídicas prestacionales de tal importancia que su provisión no pueda quedar en manos de las mayorías. De conformidad a este modelo, el interrogante acerca de cuáles son los derechos sociales fundamentales que tiene el individuo $-\mathrm{y}$ particularmente su contenido, en el caso de derechos formulados bajo el formato de principios o directrices - es un asunto de ponderación. Veamos.

De una parte, en cualquier ponderación que envuelva derechos sociales - ya sea la ocasionada por la impugnación de una medida legislativa que desarrolle un derecho fundamental social o por una que lo limite, pasando por los conflictos en sede de amparo de derechos fundamentales donde comparezca uno de los derechos en comento - se encontrará alguna libertad basada en el principio de libertad fáctica como interés que se desee superponer a él. También podrán tomar parte en el conflicto concreto algunos principios institucionales, como la competencia legislativa y la separación de poderes.

En este modelo teórico y especulativo (pues como ya mencionamos, la ponderación es una operación de razonamiento práctico) la generalizada condición estructural de los derechos sociales nos dirá muy poco acerca de las prestaciones específicas que envuelve. Con todo, sí nos ayudará a identificar cuáles facultades subjetivas puede tener un individuo justificadas en la igualdad sustancial que promueven los derechos sociales.

Robert Alexy, en VVAA. Robert Alexy, derechos sociales y ponderación, 151-162 (2009). 
En efecto, y como ejercicio general y ejemplar, ante un conflicto que implique la ponderación de un derecho social, según Alexy, habrá que considerar: (1) con qué urgencia la satisfacción de la libertad fáctica y la igualdad sustancial exigen el respeto de ese derecho, y (2) si los principios que orientan la competencia legislativa y la separación de poderes se ven afectados en una medida inferior a la satisfacción del derecho social que se privilegia ${ }^{35}$.

El telón de fondo de este prototipo de ponderación no es otro que un cuestionamiento directo a la propuesta (casi ideológica) de que existiría un conflicto irreconciliable entre los derechos sociales (prestacionales o no) y la libertad en sus diferentes dimensiones. Tal afirmación se resume en la fórmula: "a mayor igualdad menor libertad". Enunciado carente de parcialidad que toma abierto partido por un sacrificio de la igualdad en pos de la libertad. Empero, como lo sugieren a través de diversos argumentos Garzón Valdés, Prieto y Alexy, la verdadera libertad — fáctica como la denomina el autor alemán- depende del reconocimiento de derechos que se orienten a la obtención de un mínimo indispensable para hablar en propiedad de actos realmente voluntarios, igualdad sustancial según Prieto - todo con el propósito enunciado por Garzón Valdés de evitar la configuración de una sociedad indecente-. Lo interesante, más allá de las propuestas de estos autores, viene dado por el recurso a la ponderación y al aprovechamiento del escenario en que ella se desenvuelve, para probar que la exigencia de igualdad no tiene otro fin que la garantía de la libertad.

35 Robert Alexy, Teoría de los derechos fundamentales, Centro de Estudios Políticos y Constitucionales, Madrid, 454 (2008). 


\section{CONCLUSIONES Y REFLEXIONES FINALES}

El supuesto carácter prestacional de los denominados derechos sociales es uno de sus elementos más mencionados, tal vez porque, desde el punto de vista jurídico, resulta más explicativo o definidor que aquellos otros que se basan en consideraciones históricas, ideológicas o sociológicas.

Con todo, si consideramos que en estricto rigor - siguiendo a Alexy - un derecho prestacional es aquel en cuya virtud se asegura a su titular algo que, si tuviese medios económicos suficientes y se encontrase disponible en el mercado, podría adquirir por sí mismo de particulares, la idea de identificarlo automáticamente con los derechos sociales es inconveniente por diversas razones.

La identificación a priori de los derechos sociales con derechos de prestación en sentido estricto, envuelve una paradoja. En general los derechos prestacionales requieren, para ser reconocidos como tales, de una suficiente determinación de sus titulares, contenidos y sujetos pasivos, además de la asignación de una garantía jurisdiccional. Sin embargo, justamente uno de los factores de aparente debilidad de los derechos fundamentales sociales está constituido por la apretura de su supuesto de hecho, la sub-determinación de sus consecuencias jurídicas y la dificultad para dispensarles tutela judicial. Es decir, de alguna manera hablar sin más de derechos sociales como derechos prestacionales en sentido estricto importa confundir su configuración positiva real con su estatuto deseado.

En la actualidad es posible hablar de un nuevo modelo de derecho y democracia: el Estado constitucional de derecho, que es fruto de un verdadero cambio de paradigma respecto al modelo paleo-positivista del Estado legislativo de derecho y en el cual, la Constitución es plenamente fuente del derecho. Contiene normas, dispone parámetros sustantivos y procedimentales para abrogar y/o invalidar normas inferiores preconstitucionales e invalidar normas posteriores que la contravengan, y se entiende que las normas constitucionales son idóneas para disciplinar directamente no solo la organización estatal y las relaciones entre el Estado y las personas, sino también las que ocurran ente estas últimas, y son por tanto susceptibles de ser aplicadas por cualquier juez y no solo por el juez constitucional. 
Tal vez, los elementos más importantes en estas nuevas cartas fundamentales sean los principios y las directrices. Los primeros se caracterizan por estar formulados como enunciados que correlacionan siempre casos con la calificación normativa de una determinada conducta, pero de manera abierta. A diferencia de las reglas, carecen de una condición de aplicación determinada.

El reconocimiento de los derechos fundamentales sociales en las cartas fundamentales del constitucionalismo contemporáneo enfatiza la dimensión objetiva de aquellos. En cuanto que normas objetivas, las cláusulas que recogen derechos sociales vinculan a todos los poderes públicos, incluido el legislador, por lo que, en principio, nada impide que sean invocados en cualquier instancia jurisdiccional y, por supuesto, que sirvan de parámetro para el juicio de constitucionalidad.

En este contexto, autores como Luis Prieto, Robert Alexy y Ernesto Garzón Valdés sugieren diversos argumentos a favor de los derechos fundamentales sociales. Particularmente interesante resulta la propuesta de Alexy en cuanto sugiere - reconociendo el carácter de principios de los derechos fundamentales sociales- la ponderación como herramienta para no solo resolver eventuales conflictos entre derechos sociales fundamentales y libertades fundamentales, sino también como una vía para disipar la supuesta tensión entre tales clases de derechos.

El telón de fondo de este prototipo de ponderación no es otro que un cuestionamiento directo a la propuesta (casi ideológica) de que existiría un conflicto irreconciliable entre los derechos sociales (prestacionales o no) y la libertad en sus diferentes dimensiones. Tal afirmación se resume en la fórmula: "a mayor igualdad menor libertad", enunciado carente de parcialidad que toma abierto partido por un sacrificio de la igualdad en pos de la libertad. Empero, como lo sugieren a través de diversos argumentos Garzón Valdés, Prieto y Alexy, la verdadera libertad - fáctica como la denomina el autor alemán- depende del reconocimiento de derechos que se orienten a la obtención de un mínimo indispensable para hablar en propiedad de actos realmente voluntarios -igualdad sustancial, según Prieto - todo con el propósito, enunciado por Garzón Valdés de evitar la configuración de una sociedad indecente. 


\section{REFERENCIAS}

\section{Libros}

Alfonso García Figueroa, Principios y positivismo jurídico, El no positivismo principialista en las teorías de Ronald Dworkin y Robert Alexy, Centro de Estudios Políticos y Constitucionales, Madrid (1998).

José Juan Moreso, Constitución: un modelo para armar, Marcial Pons, Madrid (2009).

Luigi Ferrajoli, Derecho y razón. Teoría del garantismo penal, Trotta, Madrid (1998). , sobre los derechos fundamentales en Miguel Carbonell, ed., Teoría del neoconstitucionalismo, ensayos escogidos, 71-90, Trotta, Madrid (2007).

Gustavo Zagrebelsky, El derecho dúctil. Ley, derechos, justicia, Trotta, Madrid (2008).

Luis Prieto Sanchís, Justicia constitucional y derechos fundamentales, Trotta, Madrid (2003).

-, Ley, principios, derechos, Dikynson, Madrid (1998).

Manuel Atienza \& Juan Ruiz, Las piezas del derecho, Ariel, Barcelona (1996).

-, Para una teoría postpositivista del derecho, Palestra, Lima (2009).

Robert Alexy, Teoría de los derechos fundamentales, Centro de Estudios Políticos y Constitucionales, Madrid (2008).

Robert Alexy, en VVAA. Robert Alexy derechos sociales y ponderación, 151-162 (2009).

\section{Contribuciones en obras colectivas}

Alexei Julio Estrada, Los tribunales constitucionales y la eficacia entre particulares de los derechos fundamentales en Miguel Carbonell, ed., Teoría del neoconstitucionalismo, ensayos escogidos, 121-148, Trotta, Madrid (2007).

Ernesto Garzón Valdés, Algo más acerca del coto vedado, Doxa, No. 6, 209-213 (1989).

- Cinco consideraciones acerca de la concepción de derechos sociales de Robert Alexy, en VVAA. Robert Alexy derechos sociales y ponderación, 151-162 (2009).

Luis Prieto Sanchís, El constitucionalismo de los derechos, en Miguel Carbonell, ed., Teoría del neoconstitucionalismo, ensayos escogidos, 213-236, Trotta, Madrid (2007).

, Neoconstitucionalismo y ponderación judicial, en Miguel Carbonell, Neoconstitucionalismos, Trotta, Madrid (2005).

Manuel Aragón Reyes, La constitución como paradigma en Miguel Carbonell, ed., Teoría del neoconstitucionalismo, ensayos escogidos, 29-40, Trotta, Madrid (2007). 


\section{Artículos de revistas}

Luis Prieto Sanchís, Los derechos sociales y el principio de igualdad sustancial, Revista del Centro de Estudios Constitucionales, No. 22, 9-56 (1995).

Susana Pozzolo, Neoconstitucionalismo y especificidad de la interpretación constitucional, Doxa, No. 21, 339-353 (1998). 